\title{
BMJ Open Quality Postpartum depression screening: are we doing a competent job?
}

\author{
Sathyanarayan Sudhanthar (D) , Zile-e-huma Sheikh, Kripa Thakur
}

To cite: Sudhanthar S, Sheikh Z, Thakur K. Postpartum depression screening: are we doing a competent job? BMJ Open Quality 2019;8:e000616. doi:10.1136/ bmjoq-2018-000616

- Additional material is published online only. To view please visit the journal online (http://dx.doi.org/10.1136/ bmjoq-2018-000616).

Received 22 December 2018 Revised 6 September 2019 Accepted 30 September 2019

Check for updates

(C) Author(s) (or their employer(s)) 2019. Re-use permitted under CC BY-NC. No commercial re-use. See rights and permissions. Published by BMJ.

Pediatrics and Human Development, Michigan State University College of Human Medicine, East Lansing, Michigan, USA

Correspondence to Dr Sathyanarayan Sudhanthar; sudhanth@msu.edu

\section{ABSTRACT}

Postpartum depression (PPD) is one of the most common and severe postpartum morbidity, affecting 10\%-20\% of mothers within the first year of childbirth. The adverse effects of PPD, namely, prevention of motherbaby bonding and early cessation of breastfeeding, adversely affects infant growth and brain development. Studies have found that up to $50 \%$ of women with PPD go undiagnosed. Despite the American Academy of Pediatrics (AAP) recommendations, only a small percentage of paediatricians are currently screening for PPD. This project aimed to improve PPD screening using a validated tool to $75 \%$ in a primary care inner-city clinic serving a predominantly underserved population as per AAP recommendations. Baseline data for 40 charts of 2-month-old and 4-month-old well-child visits showed no documentation of PPD screening. The screening tool used for this project was the Edinburgh Postpartum Depression Scale (EPDS), which is a validated 10 -item screening questionnaire for PPD. Three Plan-Do-Study-Act (PDSA) cycles were implemented involving educational strategies, system-based practice improvement and stakeholder participation. Improvement seen after PDSA cycle 1 was minimal. At the end of cycle 2, 16/50 (33\%) charts had documentation of screening using EPDS. At the end of cycle 3, 33/40 (82\%) charts had EPDS documentation, an increase of $49 \%$ from cycle 2 . There were eight in total positive PPD screenings between cycles 2 and 3. These patients were provided counselling support through a social worker and referral services through the local community mental health organisation. We achieved more than our 75\% target goal for PPD screening implementation at the residency clinic, thereby increasing residents' awareness of PPD and the importance of PPD screening. Poststudy follow-up shows that screening was maintained at a higher rate but never reached $100 \%$.

\section{PROBLEM}

The project was undertaken at the Ingham County Health Department, the local county paediatric clinic serving a mostly underserved population with more than $97 \%$ of the patients covered by Medicaid. The clinic serves about 6000 children from the mid-Michigan area. The clinic serves as a primary teaching clinic for the residents of Michigan State University (MSU)-Sparrow paediatric programme, an Accreditation Council for Medical Education (ACGME) accredited programme with 24 resident providers rotating through the clinic. The residents are at different levels of training, and their patient load depends on their level of training. The clinic is supervised by faculty from the MSU paediatric departments on a rotating basis. The clinic also has a resource for one social worker and one behavioural health consultant (BHC) from the local community mental health centre. In 2010, the American Academy of Pediatrics (AAP) issued guidelines regarding postpartum depression (PPD) screen in paediatric offices. Our internal review showed variability in administering the depression scale to postpartum mothers, thereby potentially missing the diagnosis and delivery of services to many mothers of newborn children. Besides, many residents were not aware of the current recommendations regarding the PPD screen. This prompted the initiation of this project due to mainly two reasons, underutilisation of resources and not following practice guidelines as recommended by AAP.

The aim for this project was to improve screening for PPD to $75 \%$ in a primary care inner-city clinic serving predominantly underserved population by using a validated tool for all infants' visits at 2 and 4 months as per AAP recommendations.

\section{BACKGROUND}

The term PPD is changing to peripartum depression. According to the Diagnostic and Statistical Manual-V (DSM-V), PPD was removed as a separate entity and instead added the peripartum specifier to Major Depressive Disorder (MDD). To be considered as PPD, the parent should experience symptoms of MDD within pregnancy or up to 4 weeks after childbirth. ${ }^{1}$ The International Disease Classification (ICD-10) also does not identify PPD as a separate diagnosis. It classifies PPD as 'Mental and behavioural problems which cannot be classified anywhere but associated with puerperium within the first 6 weeks of child birth'. ${ }^{2}$ Postpartum blues, which results in mild irritation, mood swings, tearfulness and fatigue, has been reported in the first 10 days of childbirth. The incidence of the blues has been estimated to vary 
between $15 \%$ and $85 \%$, and though most mothers with postpartum blues do not need intensive treatment, the severity of the blues may be a predictor of developing the PPD. ${ }^{3-5}$ Even though the strict classification of PPD a with specified time limit varies from 4 to 6 weeks after child birth, PPD may manifest in mothers up to 6 months after delivery. ${ }^{67}$

It has been widely estimated that the incidence of PPD ranges between $10 \%$ and $15 \%$ in economically developed countries. ${ }^{67}$ However, the incidence across the world varies widely due to differences in cultural practices, reporting and family support. Studies have estimated PPD in Korea to be around $36 \%$ within 6 weeks after delivery and in Iran to be $34 \% .{ }^{89}$ One study by Halbriech et al estimated PPD prevalence and reporting to be anywhere from $0 \%$ to $60 \%$ and concluded that the widely cited prevalence of $10 \%-15 \%$ for PPD might not be reflective of the burden of the problem. ${ }^{10}$

Multiple studies have well documented the impact of PPD on maternal, paternal and child health. PPD has been attributed to cessation or higher odds of non-continuance of breastfeeding, erratic sleeping in moms and babies, waking up of babies during nights in the late infancy period, unsafe infant sleeping habits, maternal fatigue, children receiving fewer well-child visits at 12 months of age and not receiving recommended vaccinations by 24 months of age. ${ }^{11-14}$ Two studies have shown that mothers with PPD also did not conform to the standard child safety practices such as using car seats, using electric plug safety covers, safety latches and lowering bath water temperature. ${ }^{15-17}$

Paternal depression is estimated to be about $6 \%$ with the birth of a child and increases when there is associated PPD in the partner as well. This may also lead to separation from partner, arguments, divorce and unsafe family situations with violence, substance abuse and so on. AAP recommends screening for PPD at 1-month, 2-month and 4-month infant well visits with a standardised developmental scale. ${ }^{18}$ Many scales have been validated for depression screen. The United States Preventive Services Task Force (USPSTF) recommends using a validated tool to screen for depression in all adults aged 18 years and older as well as for PPD. It has endorsed the Edinburgh Postpartum Depression Scale (EPDS) as one validated tool for assessing PPD. ${ }^{19}$ The EPDS is a 10 question self-reported scale, with translated versions validated in many countries. The AAP has recommended a score of 10 as a cut-off for referral to resources. ${ }^{18}$

\section{MEASUREMENT}

On retrospective review of clinical charts for 1-month, 2-month and 4-month visits between 2015 and 2016 for baseline measurement, none of the 40 charts had any documentation of discussing PPD or screening for PPD. The charts were pulled randomly with the help of the Health Information Team (HIT). The sample size was chosen based on the number of newborn visits to the

\begin{tabular}{lllll}
\hline \multicolumn{3}{l}{$\begin{array}{l}\text { Table } 1 \\
\text { cycles }\end{array}$} & \multicolumn{3}{l}{ Age groups of children screened during PDSA } \\
\hline & $\begin{array}{l}\text { Baseline } \\
\text { data }\end{array}$ & PDSA1 & PDSA2 & PDSA3 \\
$\begin{array}{l}\text { 1-month well } \\
\text { visits }\end{array}$ & 10 & 12 & 15 & 13 \\
$\begin{array}{l}\text { 2-month well } \\
\text { visits }\end{array}$ & 15 & 13 & 17 & 14 \\
$\begin{array}{l}\text { 4-month well } \\
\text { visits }\end{array}$ & 15 & 15 & 18 & 13 \\
\hline \begin{tabular}{l} 
Total screened \\
\hline
\end{tabular} & 40 & 40 & 50 & 40 \\
\hline
\end{tabular}

PDSA, Plan-Do-Study-Act.

residency clinic encounters in 12 months. Moreover, anecdotal information collected from resident providers highlighted a knowledge gap regarding PPD and tools available for screening (table 1).

\section{DESIGN}

It was clear that the residency clinic had to make changes to adhere to the AAP guidelines. This was decided to be done as a Quality Improvement (QI) project based on the Plan-Do-Study-Act (PDSA) cycle. One resident was chosen to be the driving force for the project and used this project as part of the required research project at the end of the 3-year training in Paediatrics. One mentor faculty was appointed and along with the resident, formed the core team of the QI. Other stakeholders identified were remaining resident providers, core residency faculty who oversee the trainees, the clinic nursing staff and the social worker and the BHC. The core QI team met every 2-3 months to discuss logistics, survey distribution to the residents and to coordinate with clinic staff.

As the first step for the PDSA cycle, the core QI team decided to administer a 5-question survey to collect information about current PPD screening practices and to stimulate providers' interest in PPD (see online supplementary file: Survey Instrument for the Postpartum depression screen-PDSA 1). The survey was administered through an online survey platform. It was sent to all the resident providers and core-supervising physicians. Afterwards, the EPDS instrument was introduced to the clinic staff, residents and providers, and questions were answered on scoring and interpretation of the tool. A positive cut-off score of 10 was finalised, indicating a need for further interview, history and referral. Resources were also put in place in the clinic by the social worker and the BHC with the help of regional community mental health centres to help the mothers who were identified with PPD. For PDSA cycle 2, a clinic protocol was developed and disseminated to all the residents, staff and supervising providers; the protocol was modified for PDSA cycle 3. The core QI team also developed materials needed for the didactic session with resident providers, which were used in the PDSA cycle 2 (see online supplementary file: 


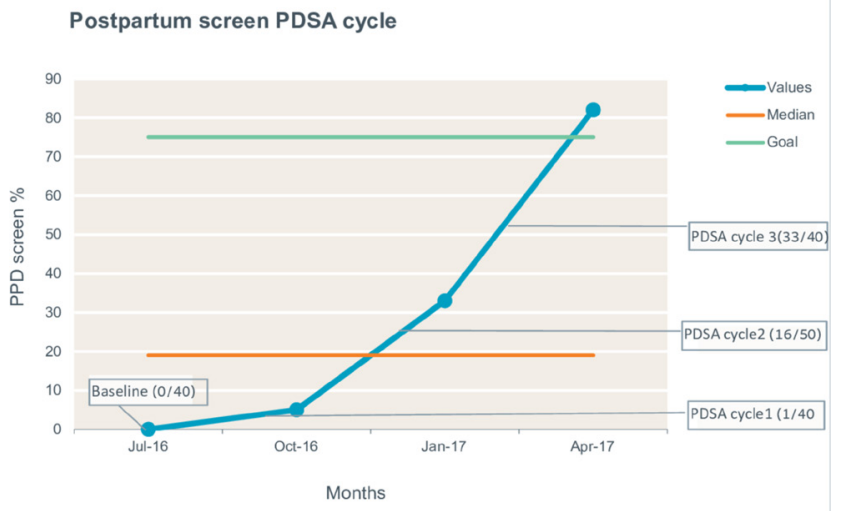

Figure 1 Illustration of run chart documenting the three PDSA cycles for the postpartum depression screening.

Final Clinic Flow Protocol for Postpartum Depression Screening).

\section{STRATEGY}

The QI project had three PDSA cycles with a 3-month interval between the cycles. As stated earlier, the goal was to increase PPD screening to $75 \%$. In the first PDSA cycle, a 5-question survey on PPD was administered to all the residents and core faculty to increase their interest in screening for PPD. The questions assessed if the healthcare provider knew the current guidelines regarding PPD if they discussed PPD with new moms if they screened with a validated screen and so on. The survey was set up in such a way that the current recommendations from the AAP were included at the end before the resident providers logged out of the survey. This cycle aimed to pique the interest of the residents and measure if that increased the rate of PPD screen during the 1-month, 2-month and 4-month well visits. In the second PDSA cycle, all residents and core faculty attended a 1-hour didactic session on PPD. The session was included as a part of the mandatory resident didactic sessions, which occurred every Thursday morning. The didactic session was delivered by the QI resident lead and covered the burden of PPD, its underdiagnosis, impact on the infant and family, the current AAP recommendations and the use of validated questionnaires such as EPDS for screening. Residents were encouraged to consider having the patients fill out the EPDS primarily at the 1-month, 2-month and 4-month visits. The QI team found that many times, the residents forgot to give out the questionnaire and thereby missed the opportunity of addressing PPD with mothers. In the third PDSA cycle, the handing out of the questionnaires was changed to the front desk staff who check in the patients. This was done due to lower than optimal rates of the PPD screen for patients in the designated age groups. The medical assistant or the nurse rooming the patient collected EPDS after the mother filled out and handed them to the provider to interpret and recommend further steps if necessary. The management of patients based on high scores was done as per the clinic protocol developed with the social worker and BHC helping the residents to identify, address and effectively manage PPD as per the AAP guidelines. The population screened were all English-speaking mothers; hence, only the English version of the EPDS was used.

\section{RESULTS}

Total $44 \%$ of the resident providers ( $n=24)$ replied, 'Never or rarely' when asked if they addressed PPD during their well-child visits. Among those who did address PPD, 75\% of the providers did during newborn and 1-month wellchild visits. Total $80 \%$ of the resident providers agreed that they did not use a validated screening tool and $94 \%$ of the resident providers were not sure of the current guidelines regarding PPD. On being asked to comment on the likely guidelines, a third of the residents accurately estimated the AAP recommendations. At the end of PDSA cycle 1 , only 1 of the 40 charts $(<5 \%)$ had documentation of administering a validated screening.

After changes made in PDSA cycle 2 with education and clinic protocol development, the PPD screening rate jumped to 33\% (16 patients were administered EPDS out of 50 patient visits) at the end of PDSA cycle 2, which showed that the combination of didactics and the reassignment of administering the questionnaire to the residents yielded a positive response though it was still less than our goal. The screening rate had improved in 3 months, but still, we were missing two out of three mothers.

After the modification of the clinic flow protocol in PDSA cycle 3, out of the 40 well-child visits, 33 had documentation of a valid screening such as the EPDS. The screening had improved to $82 \%$ in 3 months, an increase of $49 \%$ from Cycle 2.

During the 6-month timeline of PDSA cycles 2 and 3, there were eight positive screens for PPD (10\%). For those who were positive for PPD, social worker and counselling support were provided, and referral services were made through the local community mental health organisation (see figure 1).

\section{LESSONS AND LIMITATIONS}

There were many lessons learnt from this project, and one of the most important ones is that passive learning does not work for behaviour change. For example, we hoped merely sending a survey and providing the current recommendations for PPD screen at the end of the survey would at least make a modest jump in screening.

A didactic session along with skills about how to interpret the EDPS did show some promise though it was clear if a change needs to be made, the best strategy was to integrate within the system rather than relying on human behaviour as we noted in PDSA cycle 2. The resident providers very well understood the importance of PPD screening but were forgetting to administer the questionnaires. Once we made the change for the screening instrument to be administered as an integral part of the 
clinic through the front desk staff, the rate improved swiftly.

Besides, it is essential to screen and have resources available for patients to access when they have reported PPD. The QI team worked with the social worker and the BHC to develop a plan that was appropriate for our patient population with most of them classified as underserved. Of note, the PPD was positive in $10 \%$ out of the relatively small number of charts we pulled. The $10 \%$ prevalence we found in our clinic is almost the same as the national average of $10 \%-15 \%$ in the USA. It is also imperative to have a dedicated QI team to be the driving force to make everyone understand the purpose and the perceived benefits of screening. Anecdotal data gathered from our social worker, BHC, clinic staff and resident providers showed there was no impact of administering the screen on the clinic flow.

\section{CONCLUSION}

The project was an excellent experience for the QI team in terms of its ultimate objective of screening PPD early and helping the families. Throughout the project, we learnt that clinical staff is ready to make changes, but it needs to be practical and integrated. The project is easily implementable and sustainable. One-year follow-up data from May 2017 to May 2018 shows an implementation rate of 85\% (85/100 patients) and May 2018-May 2019 showed an implementation rate of $82 \%$ (82/100 patient visits). Some of the barriers are continued medical staff training due to turnover, new resident physician training in the protocol and interpretation. Clear clinic flow protocols may help in implementation, and the instrument used for screening is free to obtain and use. The challenge in providing resources for those who meet the criteria for PPD needs to be addressed early and integrated within the clinic protocol for this to be a success. This project succeeded in increasing the screening and identified at-risk mothers for PPD and helped them make a connection with community resources. This, in turn, likely influences the family dynamics, child-mother interaction and child safety in a positive manner. The plan is to continue administering the EPDS and improve the screening to all mothers by improving the rate to $100 \%$ with the help of resident and clinic staff continued buy-in. As recommended by various professional organisations, PPD screening can be implemented successfully within a primary care practice with minimal resources and help new mothers and families in need.

Acknowledgements We are thankful to the medical staff, social worker and behavioural health consultant of the Cedar Peds Residency Clinic for their contribution to make this project a success. We also wish to thank and acknowledge Dr Brian Mavis Ph.D., Professor and past Director of CHM Learning Academy, Michigan State University for reviewing, editing and providing vital inputs to this manuscript.

Contributors SS planned the study and created a first draft of the manuscript. ZS was involved in the operational part of the project including collecting data over the three cycles and involved in the strategy, design and result parts of the manuscript.
SS and KT were involved in designing the project and overall manuscript. All authors agree on the final version of the manuscript draft.

Funding The authors have not declared a specific grant for this research from any funding agency in the public, commercial or not-for-profit sectors.

Competing interests None declared.

Patient consent for publication Not required.

Provenance and peer review Not commissioned; externally peer reviewed.

Open access This is an open access article distributed in accordance with the Creative Commons Attribution Non Commercial (CC BY-NC 4.0) license, which permits others to distribute, remix, adapt, build upon this work non-commercially, and license their derivative works on different terms, provided the original work is properly cited, appropriate credit is given, any changes made indicated, and the use is non-commercial. See: http://creativecommons.org/licenses/by-nc/4.0/.

ORCID iD

Sathyanarayan Sudhanthar http://orcid.org/0000-0001-9586-1759

\section{REFERENCES}

1 American Psychiatric Association. Diagnostic and statistical manual of mental disorders. 5th ed., (DSM-5). Washington, DC: American Psychiatric Publishing, 2013.

2 World Health Organization. International statistical classification of diseases and related health problems, 10th revision, 2016.

3 Henshaw C. Mood disturbance in the early puerperium: a review. Arch Womens Ment Health 2003;6:s33-42.

4 Heron J, Craddock N, Jones I. Postnatal euphoria: are 'the highs' an indicator of bipolarity? Bipolar Disord 2005;7:103-10.

5 Reck C, Stehle E, Reinig K, et al. Maternity blues as a predictor of DSM-IV depression and anxiety disorders in the first three months postpartum. J Affect Disord 2009;113:77-87.

6 Pearlstein T, Howard M, Salisbury A, et al. Postpartum depression. Am J Obstet Gynecol 2009;200:357-64.

7 Davé S, Petersen I, Sherr L, et al. Incidence of maternal and paternal depression in primary care: a cohort study using a primary care database. Arch Pediatr Adolesc Med 2010;164:1038-44.

8 Youn JH, Jeong IS. Predictors of postpartum depression: prospective cohort study. J Korean Acad Nurs 2013;43:225-35.

9 Taherifard P, Delpisheh A, Shirali R, et al. Socioeconomic, psychiatric and materiality determinants and risk of postpartum depression in border city of Ilam, Western Iran. Depress Res Treat 2013;2013:1-7.

10 Halbreich U, Karkun S. Cross-cultural and social diversity of prevalence of postpartum depression and depressive symptoms. $J$ Affect Disord 2006;91:97-111.

11 Dennis CL, Creedy D. Psychosocial and psychological interventions for preventing postpartum depression. Cochrane Database Syst Rev 2004:CD001134.

12 Dennis C-L, McQueen K. Does maternal postpartum depressive symptomatology influence infant feeding outcomes? Acta Paediatr 2007;96:590-4.

13 Dennis C-L, Ross L. Relationships among infant sleep patterns, maternal fatigue, and development of depressive symptomatology. Birth 2005;32:187-93.

14 Minkovitz CSet al. Maternal depressive symptoms and children's receipt of health care in the first 3 years of life. Pediatrics 2005;115:306-14.

15 McLearn KT, Minkovitz CS, Strobino DM, et al. Maternal depressive symptoms at 2 to 4 months post partum and early parenting practices. Arch Pediatr Adolesc Med 2006;160:279-84.

16 McLearn KT, Minkovitz CS, Strobino DM, et al. The timing of maternal depressive symptoms and mothers' parenting practices with young children: implications for pediatric practice. Pediatrics 2006;118:e174-82.

17 Mulvaney C, Kendrick D. Do maternal depressive symptoms, stress and a lack of social support influence whether mothers living in deprived circumstances adopt safety practices for the prevention of childhood injury? Child Care Health Dev 2006;32:311-9.

18 Earls MF, Committee on Psychosocial Aspects of Child and Family Health American Academy of Pediatrics. Incorporating recognition and management of perinatal and postpartum depression into pediatric practice. Pediatrics 2010;126:1032-9.

19 Cox JL, Holden JM, Sagovsky R. Detection of postnatal depression. development of the 10-item Edinburgh postnatal depression scale. Br J Psychiatry 1987;150:782-6. 